\title{
A GENERALIZED MODEL OF THE DEVELOPMENT OF NONISOTHERMAL, AXISYMMETRIC FREE JETS
}

\author{
KUNIO KATAOKA, HiROYUKI SHUNDOH AND \\ HrTOSHI MATSUO \\ Department of Chemical Engineering, Kobe University, Kobe 657
}

\begin{abstract}
A generalized model for axisymmetric free jets which allows for change in fluid density with temperature is proposed by experiment. Experimental work was carried out to obtain data on jet velocity and temperature variations over a wide range of jet-to-ambient fluid density ratio (i.e. the initial density ratio) by use of nonisothermal free jets of burned gas exhausting into quiescent air as well as isothermal free jets of $\mathrm{CO}_{2}$-air mixture into air. Core lengths of velocity and temperature can be correlated as a function of initial density ratio and jet Reynolds number. The radial profiles of velocity and temperature in the fully-developed region can be expressed by the isothermal formulation using the corresponding jet half-radii as the characteristic radial distance. The jet half-radii and centerline decays can be described by new characteristic streamwise coordinates which take into account the effects of core length and initial density ratio. If the initial condition at the nozzle exit is specified, the present model can determine the radial distributions of velocity and temperature at any axial position.
\end{abstract}

\section{Introduction}

Prediction of velocity and temperature variations in nonisothermal turbulent free jets at various temperature levels is of great importance in the design of heat transfer equipment dealing with hot gases, such as furnaces, dryers, and boilers. For example, knowledge of the axial variations of velocity and temperature is necessary to estimate the rates of impingement heat transfer around the stagnation point of an impinging jet. Although turbulent jets have been extensively studied, most existing experimental data are for unheated or incompressible jets. The jet development of high-temperature gas exhausting into quiescent low-temperature gas is greatly influenced by the temperature or density difference between the jet and the ambient receiving medium.

Some of the previous work on compressible turbulent jets, especially those issuing into quiescent gas, will be briefly reviewed to clarify the purpose of this paper. The effect of large density difference was first investigated by Corrsin and Uberoi ${ }^{11}$, who measured velocity and temperature variations in a jet of heated air exhausting into quiescent air (up to the initial density ratio $\rho_{\infty} / \rho_{0}=2$ ). They found that a decrease of density with respect to that of the receiving medium caused an increase in the decay and spreading rates of the jet. Several theoretical approaches have been developed for prediction of turbulent mix-

Received April 2, 1981. Correspondence concerning this article should be addressed to K. Kataoka. H. Shundoh is now at Fuji Electric Co., Ltd., Yokosuka 240-01. H. Matsuo is at Mitsubishi Kasei Co., Ltd., Kurashiki 712. ing in relatively high-speed compressible jets: the turbulent mixing coefficient model due to Warren ${ }^{10}$ and Donaldson and $\mathrm{Gray}^{2}$, the dynamic eddy transfer coefficient model due to Ferri et al. ${ }^{4}$, Kleinstein ${ }^{6)}$, Tomich and Weger ${ }^{8)}$, Witze ${ }^{12)}$, and Kataoka and Takami $^{5}$, and the extended Reichardt free-mixing theory due to Sforza and Mons ${ }^{7}$. All these theoretical approaches are ineffective without the simplifying assumption of local eddy diffusivity variation with density. Experimental work ${ }^{2,5,7,10)}$ has also been done to support those respective theories, but has been restricted to small changes in the initial density ratio. The present author and coworkers ${ }^{5 /}$ successfully extended Prandtl's eddy diffusivity model to nonisothermal turbulent jets by taking into account the change in volume of traveling eddies due to large thermal gradients. They also obtained experimentally a model of dynamic eddy transfer coefficients for momentum, heat, and mass transfer in turbulent jets of hightemperature burned gas issuing into quiescent air $\left(\rho_{\infty} / \rho_{0}=5.1\right)$. As a result, it has been found that the dynamic eddy transfer coefficients for heat and mass transfer are about 1.5 times as large as for momentum transfer. However, the expressions describing the decay and spreading of nonisothermal jets remain to be improved with relation to the effect of the initial density ratio. Still, few experimental data are available for the heated jet, especially at high temperatures.

It should be noted that Thring ${ }^{\theta)}$, Kleinstein ${ }^{6)}$, Wilson $^{11)}$, and Sforza ${ }^{7)}$ proposed $\sqrt{\rho_{\infty} / \rho_{o}} Z / D$ as the characteristic streamwise coordinate. However, they 


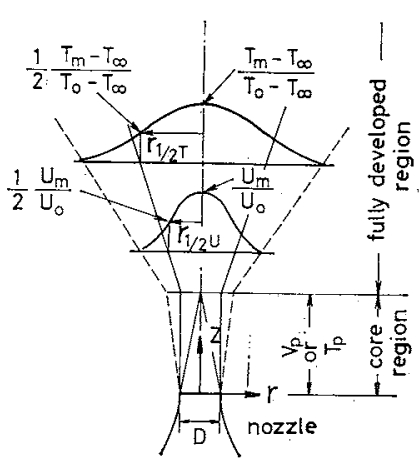

Fig. 1 Schematic diagram of nonisothermal free jet and coordinates used

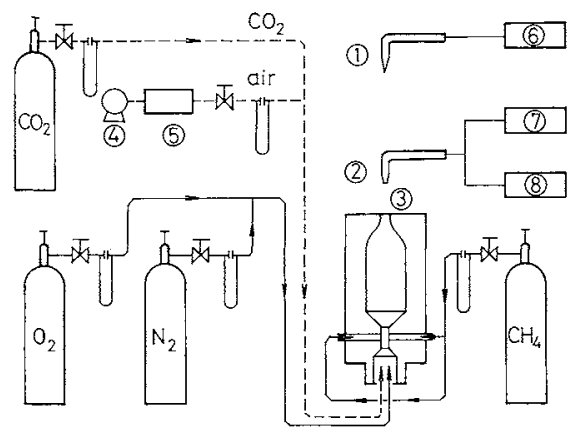

(1) $\phi 0.1 \mathrm{~mm} \mathrm{Pt} / \mathrm{PtRh}$ thermocouple

(2) $\phi 1.4 \mathrm{~mm}$ OD quartz total head tube

(3) $\phi 10.0 \mathrm{~mm}$ convergent nozzle

(4) air compressor

(5) water eliminator

(6) analog recorder

(7) Göttingen manometer

(8) gas chromatograph

Fig. 2 General arrangement of experimental apparatus

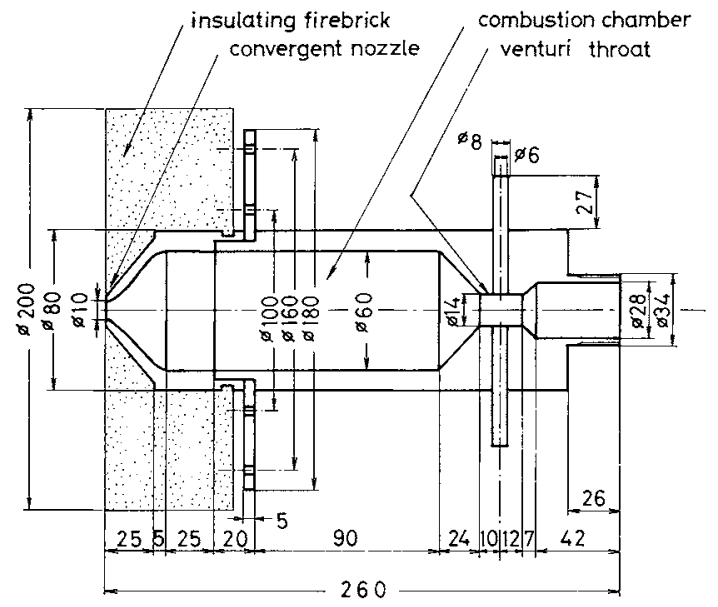

Fig. 3 Nozzle assembly

did not consider the effect of $\rho_{\infty} / \rho_{o}$ on core length, except that Sforza adopted the virtual origin. At the present stage, it is more fruitful to establish a generalized model describing jet development over a wide range of initial density ratio from the practical viewpoint. This research was limited to a low Mach number range (Mach number $<0.1$ ), where the effect of compression due to velocity change was negligibly small. Figure 1 is a schematic diagram of the nonisothermal free jet showing the coordinate system used.

\section{Experimental Apparatus and Procedure}

The experimental apparatus consists of a stainless steel nozzle assembly, which provides hot free jets at various temperatures by combustion of methane gas, and a measuring system with a traversing device for Pt-PtRh thermocouple and total-head tube. Figure 2 shows the general arrangement of the apparatus. The nozzle assembly is shown in Fig. 3. It consists of a burner having a venturi throat for intimately mixing methane gas with an oxidizing stream, a 60 mm-ID combustion chamber with a straight section $135 \mathrm{~mm}$ long, and a $10 \mathrm{~mm}$-ID convergent nozzle with a contraction ratio of $1 / 36$. The inside surface of the nozzle was carefully machined so as to make exit velocity profiles as uniform as possible. The nozzle outlet was also arranged flush with, and central to, the horizontal surface of an insulating firebrick not only to provide exit temperature profiles as uniform as possible, but also to prevent entrainment of the ambient air from behind the nozzle. Therefore, this nozzle belongs to the so-called blunt-edged nozzle category $^{2}$. Methane, oxygen, and nitrogen flows are separately regulated with needle-valve flow controllers to maintain accurate jet conditions. Thereafter, the ratio of molar flow rates is kept at $\mathrm{CH}_{4}: \mathrm{O}_{2}$ : $\mathrm{N}_{2}=1: 3.3: 5.3$ to make the nitrogen/oxygen ratio of the burned gas equal to that of the ambient air. For simplicity, fluid density and viscosity are determined from those of air since the average molecular weight of the burned gas is approximately equal to that of air. The hot gas produced in the combustion chamber is exhausted vertically upward from the nozzle into quiescent ambient air at room temperature. It has. been confirmed by means of gas chromatograph that no burning mixture issues from the nozzle, owing to complete combustion. In the same manner as in the previous study ${ }^{5}$, it can be confirmed that the effect of free convection due to large temperature differences between the jet and the ambient air is negligibly small except in the outer intermittent region. The gas temperature is measured directly by a $0.1 \mathrm{~mm}$-diameter Pt-PtRh thermocouple. The error due to the thermal effect of radiation has been confirmed to be within $2 \%$ by comparison with a suction thermometer of $0.2 \mathrm{~mm}$ diameter Pt-PtRh thermocouple. The dynamic pressure is measured by a miniature total-head tube of $1.4 \mathrm{~mm}$-OD quartz tube. Thereafter, the gas velocity is calculated from the measured dynamic pressure, taking into account the density change with the aid of the measured gas temperature. These probes are movable to any position by means of a traversing 
Table 1 Experimental conditions

\begin{tabular}{|c|c|c|c|}
\hline \multicolumn{4}{|l|}{ Heated } \\
\hline$U_{0}$ & 7.4 & -22.7 & {$[\mathrm{~m} / \mathrm{s}]$} \\
\hline$T_{o}$ & 823 & -1530 & {$[\mathrm{~K}]$} \\
\hline$T_{\infty}$ & 288.2 & -302.2 & {$[\mathrm{~K}]$} \\
\hline$\rho_{o}$ & 0.231 & 0.429 & {$\left[\mathrm{~kg} / \mathrm{m}^{3}\right]$} \\
\hline$\rho_{\infty}$ & 1.17 & 1.23 & {$\left[\mathrm{~kg} / \mathrm{m}^{3}\right]$} \\
\hline$\rho_{\infty} / \rho_{0}$ & 2.73 & 5.26 & [-] \\
\hline$R e_{0}$ & 857 & -1810 & {$[-]$} \\
\hline \multicolumn{4}{|c|}{ Unheated $\mathrm{CO}_{2} /$ air and air/air jets } \\
\hline$U_{o}$ & 5.76 & -12.55 & {$[\mathrm{~m} / \mathrm{s}]$} \\
\hline$T_{\infty}=T_{o}$ & 286.7 & -297.2 & {$[\mathrm{~K}]$} \\
\hline$X_{o}$ & 0 & $-\quad 1.0$ & {$[-]$} \\
\hline$\rho_{\infty} / \rho_{0}$ & 0.66 & 1.0 & {$[-]$} \\
\hline$R e_{0}$ & 4900 & -8300 & {$[-]$} \\
\hline
\end{tabular}

device with accurate lead screws that allows their locations to be determined with an accuracy of $0.1 \mathrm{~mm}$ or better. At high temperatures, the jet Reynolds number cannot be made very high owing to the high viscosity and the low density of the burned gas. The experimental conditions are limited to a narrow range of jet Reynolds number to maintain flame stability at the burner section. As will be pointed out later, the present result is in satisfactory agreement with the previous investigations ${ }^{1,7,10,11)}$ done at higher Reynolds numbers and lower initial-density ratios if the generalized model proposed here is adopted. The isothermal jet experiment is also conducted around $\rho_{\infty} / \rho_{o}=1$ by exhausting gas mixtures of $\mathrm{CO}_{2}$ and air into air from the same nozzle. The gas composition is measured by means of a gas chromatograph, using the total-head tube as a sampling probe. Experimental conditions are listed in Table $\mathbf{1}$.

\section{Experimental Results and Discussion}

This flow system should have $U_{m}, T_{m}-T_{\infty}$ and $r_{1 / 2 U}, r_{1 / 2 T}$ as the characteristic variables. It can be considered that the total momentum and enthalpy fluxes are respectively conserved:

$$
\begin{gathered}
\int_{0}^{\infty} \rho U^{2} 2 \pi r d r \simeq{ }_{4}^{\pi} \rho_{0} U_{0}^{2} D^{2} \\
\int_{0}^{\infty} \rho C p\left(T-T_{\infty}\right) U 2 \pi r d r \simeq{ }_{4}^{\pi} \rho_{0} C p_{o}\left(T_{o}-T_{\infty}\right) U_{o} D^{2}
\end{gathered}
$$

Thring and Newby ${ }^{9}$ deduced the following dimensional relations by applying the similarity assumption to the above equations:

$$
\begin{gathered}
U_{o} \simeq \sqrt{\frac{\rho_{\infty} Z}{\rho_{0} D}} \\
U_{m} \\
T_{o}-T_{\infty} \simeq \sqrt{\rho_{\infty} Z} \\
T_{m}-T_{\infty} D
\end{gathered}
$$

Still the core lengths $V_{p}, T_{p}$ or the corresponding virtual origins remain to be considered for the axial variations of velocity and temperature.

As shown in the previous paper ${ }^{5}$, the following jet characteristics have been also confirmed for the pres-

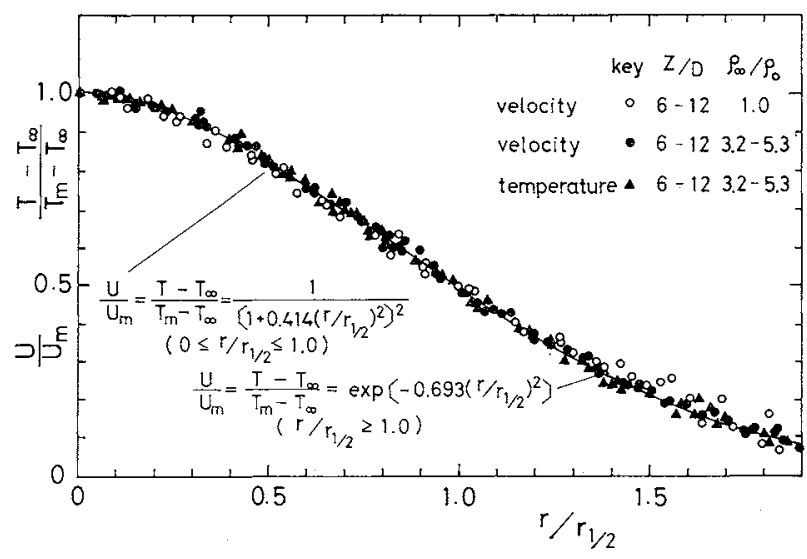

Fig. 4 Radial distribution of normalized velocity and temperature

ent geometry of the nozzle assembly: (1) the initial radial distributions of velocity and temperature are uniform over about $90 \%$ of the cross-section of the nozzle exit; (2) the radial profiles of velocity and temperature are respectively similar at various axial positions in the fully developed region and can be well described by the equations shown below.

$$
\begin{aligned}
& \left\{\begin{array}{c}
U / U_{m}=\left[1+0.414\left(r / r_{1 / 2 U}\right)^{2}\right]^{-2} \\
\quad 0 \leq r / r_{1 / 2} \leq 1 \\
\left(T-T_{\infty}\right) /\left(T_{m}-T_{\infty}\right)=\left[1+0.414\left(r / r_{1 / 2 T}\right)^{2}\right]^{-2}
\end{array}\right. \\
& \left\{\begin{array}{rr}
U / U_{m}=\exp \left[-0.693\left(r / r_{1 / 2 U}\right)^{2}\right] \\
r / r_{1 / 2} \geq 1 \\
\left(T-T_{\infty}\right) /\left(T_{m}-T_{\infty}\right)=\exp \left[-0.693\left(r / r_{1 / 2 T}\right)^{2}\right]
\end{array}\right.
\end{aligned}
$$

Figure 4 shows the similar radial distributions of the normalized velocity and temperature. These equations are mathematically similar in form to those for unheated or isothermal jets. The only difference is in the variation of the jet half-radii with temperature.

The axial variations of centerline quantities and jet half-radii are shown in Figs. 5 to 8. It can be seen from these figures (1) that the core lengths decrease with initial density ratio, (2) that the jet half-radii and the reciprocals of the centerline quantities vary linearly in the fully developed region, and (3) that the decay rate of the centerline quantities and the spreading rate of the jet widths increase with initial density ratio. Hence the first step in analyzing the jet development is to correlate the core lengths at various temperature levels. Core lengths were determined both from the centerline decay curves and from the jet spreading curves. They are coincident with each other within experimental error $(20 \%)$.

The core lengths for velocity and temperature are well correlated by

$$
\begin{aligned}
& V_{p} / D=2.82\left(\rho_{\infty} / \rho_{0}\right)^{-0.29} R e_{0}^{0.07} \\
& T_{p} / D=3.80\left(\rho_{\infty} / \rho_{o}\right)^{-0.45} R e_{0}^{0.03}
\end{aligned}
$$

As shown in Figs. 9 and 10, these equations obtained 


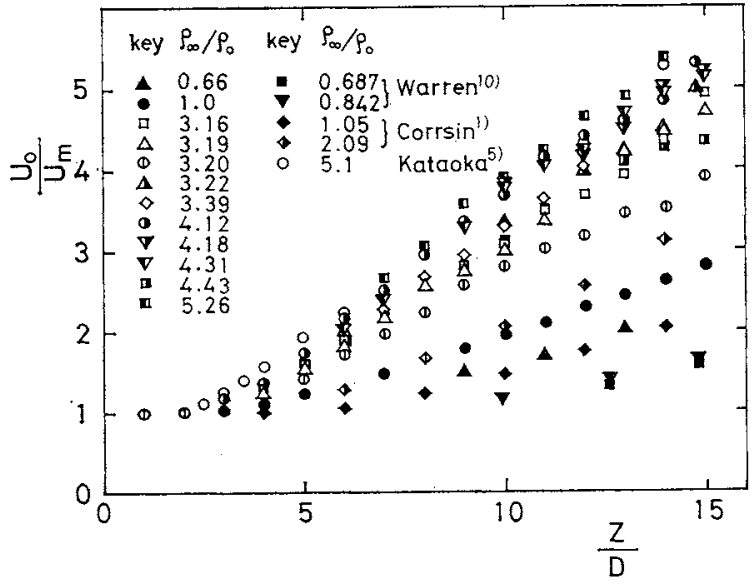

Fig. 5 Axial decay of centerline velocity

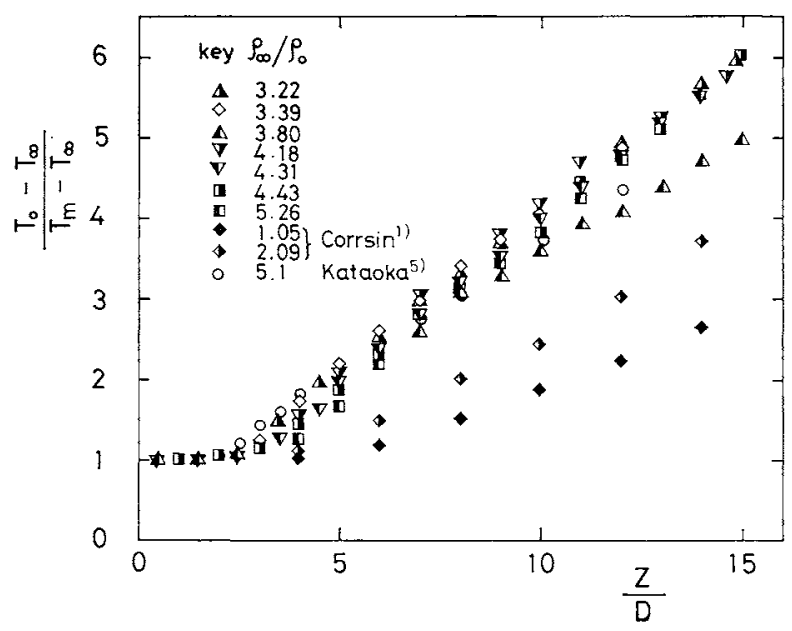

Fig. 6 Axial decay of centerline temperature

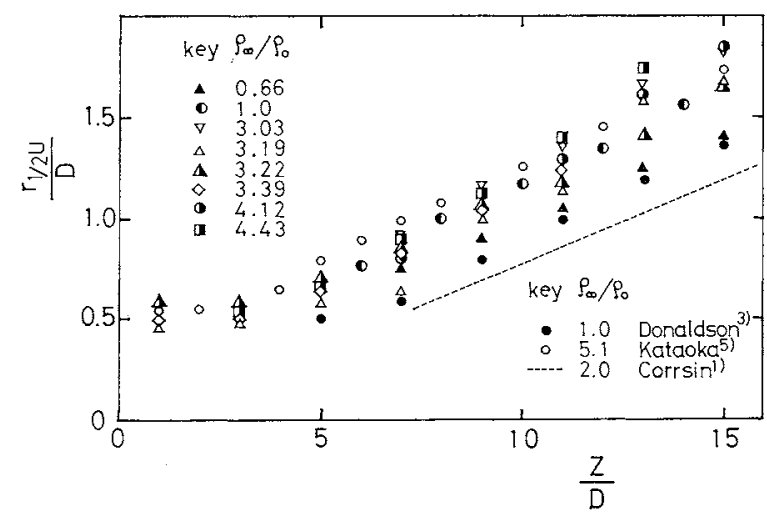

Fig. 7 Axial variation of jet half-radius for velocity

by the method of least squares hold within $20 \%$ error over a wide range $\rho_{\infty} / \rho_{o}=0.66-5.3$ including the other investigations ${ }^{1,3,11\}}$ done at higher jet Reynolds numbers. As the jet Reynolds number increases, the core lengths tend to increase slightly. The trend is similar to that of Witze's model ${ }^{12)}$ for the compressible jet. The core length is shorter for temperature than for velocity. This is attributable to the fact that the dynamic eddy transfer coefficient for

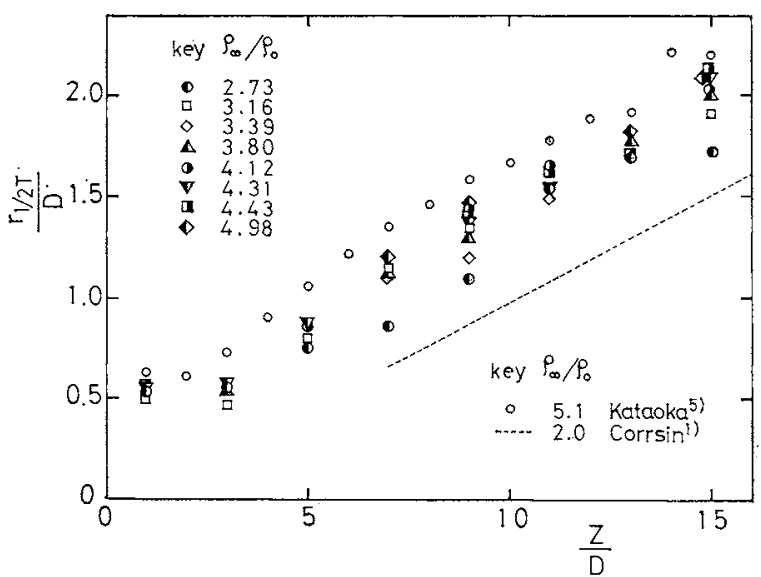

Fig. 8 Axial variation of jet half-radius for temperature

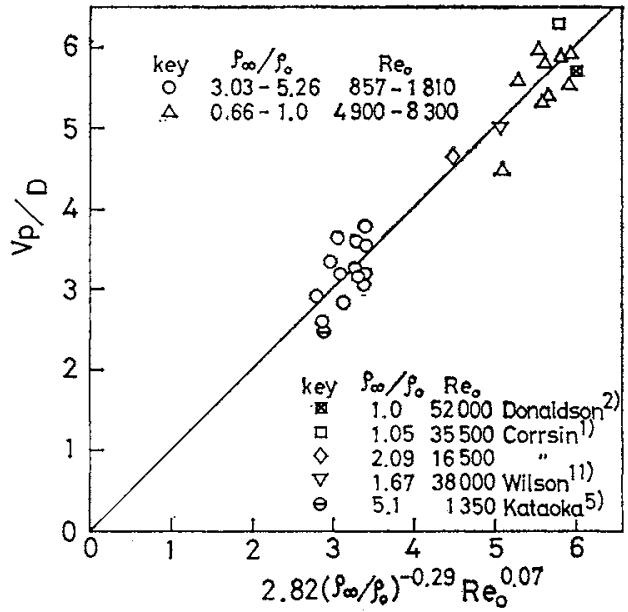

Fig. 9 Correlation of velocity core lengths

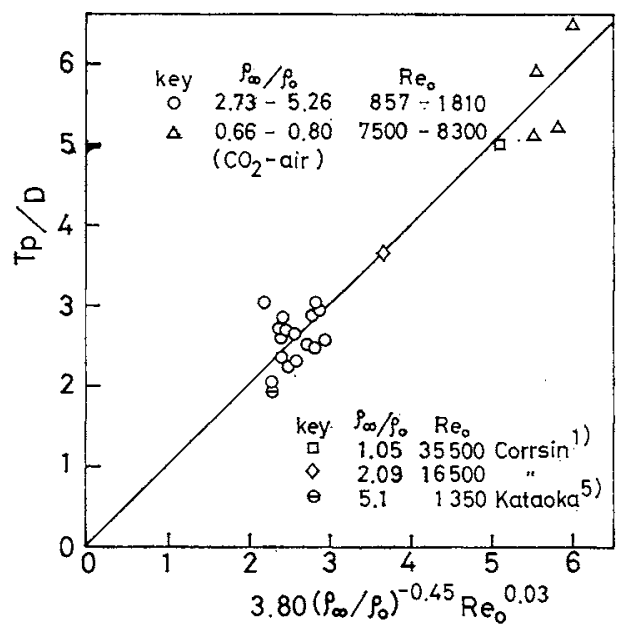

Fig. 10 Correlation of temperature core lengths

heat is larger than that for momentum in the turbulent shear layer around the core region ${ }^{5}$.

Then the centerline decays are well correlated with the characteristic streamwise coordinate, as shown in Figs. 11 and 12. The curve-fit equations are

$$
U_{o} / U_{m}=0.16\left(\rho_{\infty} / \rho_{o}\right)^{1 / 2}\left(Z-V_{p}\right) / D+1.0
$$


Fig. 11 Correlation of centerline velocities

Fig. 12 Correlation of centerline temperatures
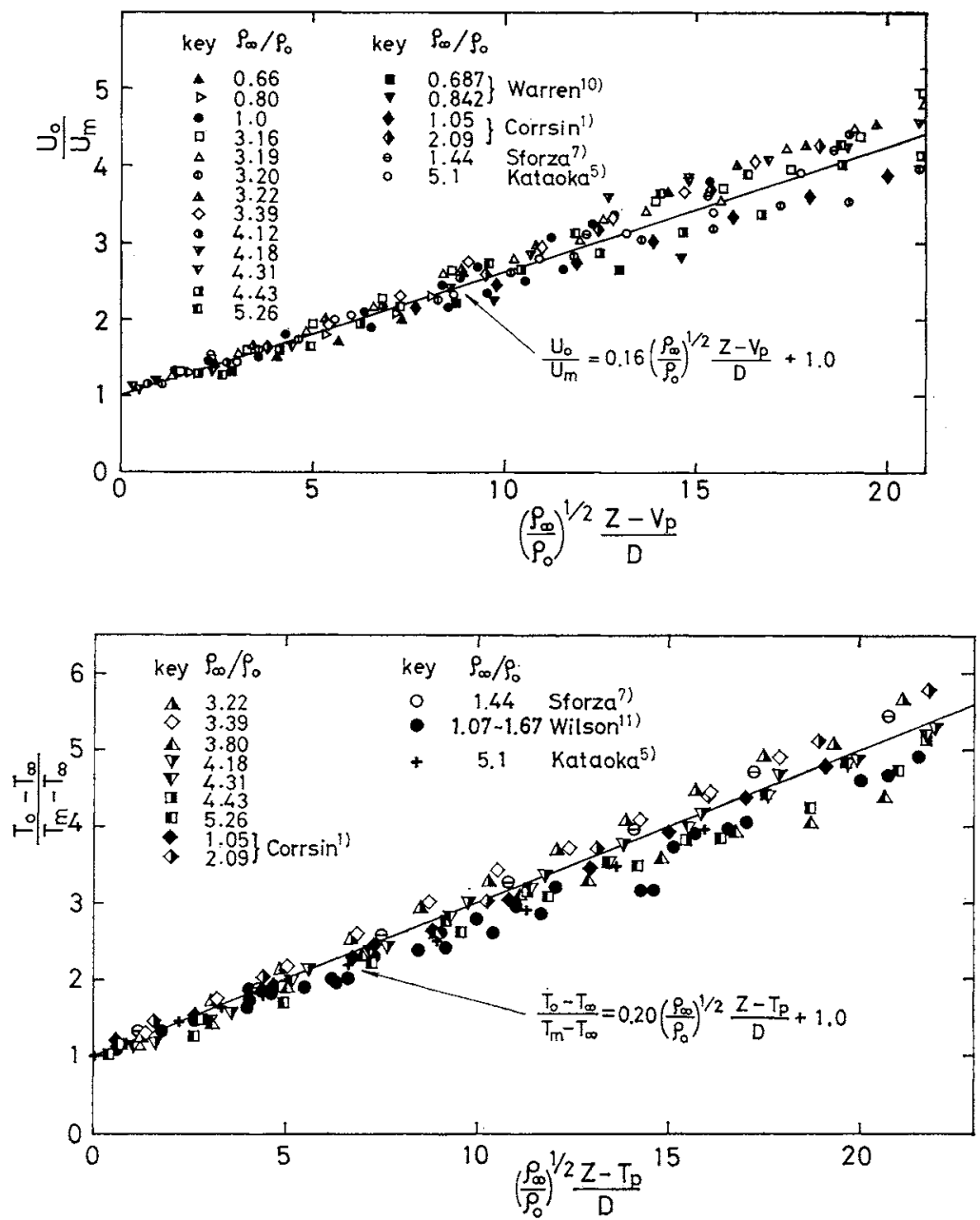

$$
\left(T_{o}-T_{\infty}\right) /\left(T_{m}-T_{\infty}\right)=0.20\left(\rho_{\infty} / \rho_{o}\right)^{1 / 2}\left(Z-T_{p}\right) / D+1.0
$$

Here the centerline decay of $\mathrm{CO}_{2}$ concentration is also included in Eq. (10). The exponent of the initial density ratio is coincident with that deduced by Thring $^{9)}$ and Kleinstein ${ }^{8}$. The intercept should have a value of 1.0 from the definition of core lengths. These equations can also describe the other investigations done at higher Reynolds numbers within $20 \%$ error, except the temperature decay data by Wilson and Danckwerts ${ }^{11}$. The streamwise coordinate is similar to that proposed by Wilson ${ }^{11}$ and Sforza ${ }^{7)}$. The only difference is that the present authors adopted core length in place of virtual origin because core length correlations are more useful from the practical viewpoint. Little work has been done on the effect of initial density ratio on virtual origin.

Figures 13 and 14 show correlations of jet halfradii for velocity and temperature. The curve-fit equations are

$$
\begin{aligned}
& r_{1 / 2 U} / D=0.09\left(\rho_{\infty} / \rho_{o}\right)^{0.1}\left(Z-V_{p}\right) / D+0.5 \\
& r_{1 / 2 T} / D=0.08\left(\rho_{\infty} / \rho_{0}\right)^{1 / 3}\left(Z-T_{p}\right) / D+0.5
\end{aligned}
$$

The intercept should have a value of 0.5 from the definition of core lengths. These equations can de-

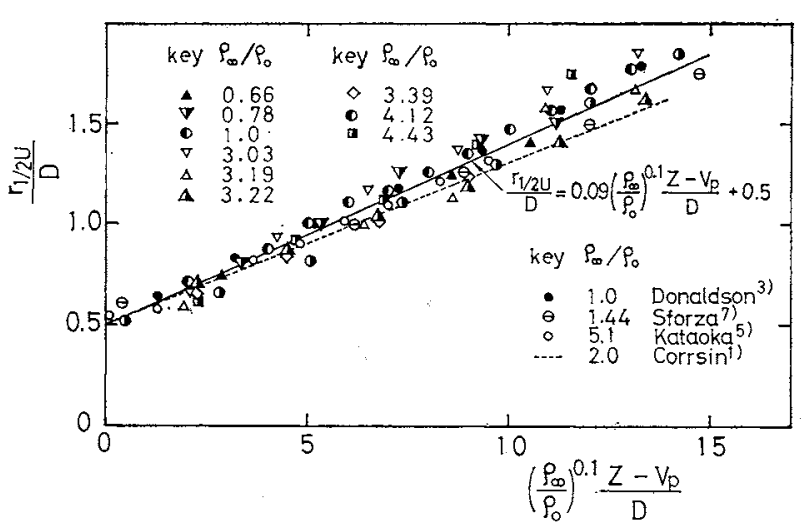

Fig. 13 Correlation of jet half-radii for velocity

scribe the other investigations ${ }^{1,3,7)}$ done at higher Reynolds numbers within $15 \%$ error. As distinct from Sforza's result, the streamwise coordinate proposed here is a slight function of the initial density ratio. This indicates that the perfect similarity assumed in the isothermal model does not hold strictly for the nonisothermal jet. Equations (7) and (8) in the previous paper ${ }^{5)}$ should be replaced by Eqs. (9) to (12) of the present paper because this correction does not influence the remaining part of the previous paper. 


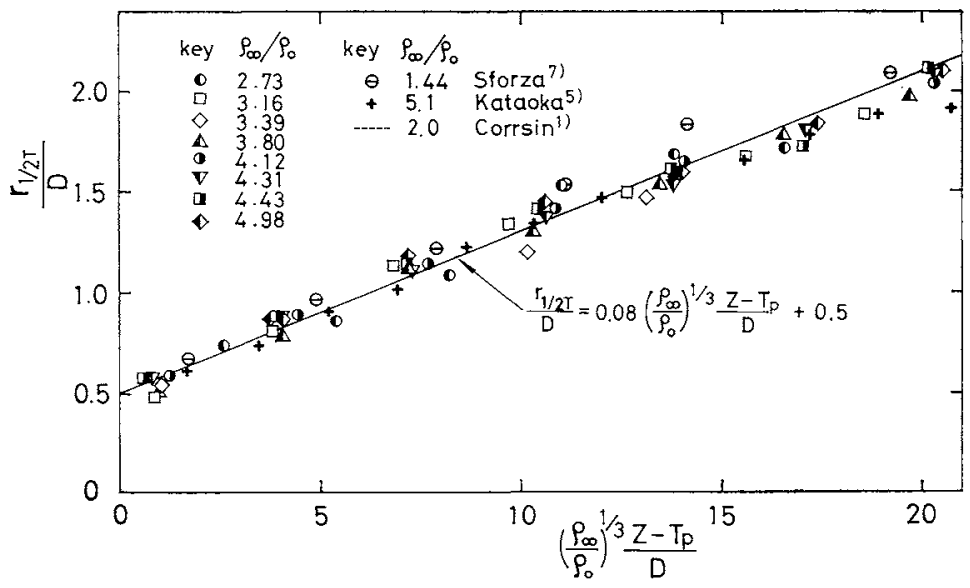

Fig. 14 Correlation of jet half-radii for temperature

A generalized model has been established. Velocity and temperature profiles at any axial position in nonisothermal free jets can be estimated at various temperature levels by the following steps:

(1) Calculate the core lengths $V_{p}, T_{p}$ by Eqs. (7) and (8).

(2) Calculate the centerline quantities $U_{m}, T_{m}$ by Eqs. (9) and (10).

(3) Calculate the half-radii $r_{1 / 2 U}, r_{1 / 2 T}$ by Eqs. (11) and (12).

(4) Then calculate the radial profiles at any axial position by Eqs. (5) and (6).

\section{Conclusions}

A generalized model has been established by experiment for prediction of the velocity and temperature variations in nonisothermal free jets at various temperature levels. The core lengths are well correlated in terms of initial density ratio and jet Reynolds number. The axial variations of the centerline quantities and jet half-radii are well correlated by new characteristic streamwise coordinates, taking into account the effects of the core length and the initial density ratio. The radial variations of normalized velocity and temperature are well correlated by dimensionless radial coordinates based on the jet halfradii. If the initial density ratio and the jet Reynolds number are specified at the nozzle exit, the distributions of velocity and temperature can be determined at any position by the proposed model.

\section{Nomenclature}

$\begin{array}{ll}C p & =\text { heat capacity } \\ D & =\text { convergent nozzle diameter } \\ r & =\text { radial distance from jet axis } \\ \operatorname{Re}_{0} & =U_{0} D / \nu_{0}, \text { jet Reynolds number }\end{array}$

$\begin{array}{llr}T & =\text { temperature } & {[\mathrm{K}]} \\ T_{p} & =\text { temperature core length } & {[\mathrm{m}]} \\ U & =\text { axial velocity } & {[\mathrm{m} / \mathrm{s}]} \\ V_{p} & =\text { velocity core length } & {[\mathrm{m}]} \\ X & =\text { mole fraction of } \mathrm{CO}_{2} \text { in } \mathrm{CO}_{2} \text {-air mixture } & [-]] \\ Z & =\text { axial distance from nozzle exit } & {[\mathrm{m}]} \\ \nu & & \\ \rho & =\text { kinematic viscosity } & {\left[\mathrm{m}^{2} / \mathrm{s}\right]} \\ & =\text { density } & {\left[\mathrm{kg} / \mathrm{m}^{3}\right]}\end{array}$

〈Subscripts〉

$$
\begin{array}{ll}
m & =\text { quantity on jet axis } \\
o & =\text { quantity at nozzle exit } \\
1 / 2 & =\text { position within jet where property is } \\
& \text { one-half of centerline property } \\
1 / 2 T & =\text { position where } T-T_{\infty}=\left(T_{m}-T_{\infty}\right) / 2 \\
1 / 2 U & =\text { position where } U=U_{m} / 2 \\
\infty & =\text { quantity in ambient fluid }
\end{array}
$$

\section{Literature Cited}

1) Corrsin, S., and M. S. Uberoi: NACA TN 1865 (1949).

2) Donaldson, C. duP., and K. E. Gray: AIAA J., 4, 2017 (1966).

3) Donaldson, C. duP., R. S. Snedeker, and D. P. Margolis: J. Fluid Mech., 45, 477 (1971).

4) Ferri, A., P. A. Libby, and V. Zakkay: 3rd ICAS Conference, Stockholm (1962).

5) Kataoka, K. and T. Takami: AIChE J., 23, 889 (1977).

6) Kleinstein, G.: J. Spacecr. Rockets, 1, 403 (1964).

7) Sforza, P. M. and R. F. Mons: Int. J. Heat Mass Transfer, 21, 371 (1978).

8) Tomich, J. F. and E. Weger: AlChE J., 13, 948 (1967).

9) Thring, M. W. and M. P. Newby: Fourth Symposium on Combustion, p. 789 (1953).

10) Warren, W. R.: Ph. D. thesis, Princeton Univ., Princeton, N. J. (1957).

11) Wilson, R. A. M. and P. V. Danckwerts: Chem. Eng. Sci., 19, 885 (1964).

12) Witze, P. O.: AIAA J., 12, 417 (1974).

(A part of this paper was presented at the 45th Annual Meeting of The Soc. of Chem. Engrs., Japan, at Osaka, April 3, 1980.) 